\title{
Second Language Speech Learning
}

Zoe Handley, Department of Education, University of Oxford

My research focuses on perceptual approaches to second language speech learning, or pronunciation training. Two main approaches to perceptual training have been investigated in the literature: (1) perceptual fading in which learners are presented stimuli from opposite ends of a synthetic continuum in which the differences between the members of the non-native phonemic contrast have been exaggerated (Jamieson and Morosan, 1986), and (2) High-Variability Pronunciation Training (HVPT) in which learners are presented minimal pairs containing the nonnative phonemic contrasts in forced-choice identification format (Logan et al., 1991). While these techniques, in particular HVPT, have been demonstrated to be effective in lab-based studies, they typically involve just one simple task, e.g. forced-choice identification, which the learner is required to repeat ad nauseum. To ensure that learners persist with training when a wider variety of phonemic contrasts are to be trained, I believe that a greater variety of tasks is required. In this poster, I present an experiment which compared two versions of HVPT, one employing an identification task and one employing a discrimination task. Future research will explore a wider variety of tasks in combination with the use of synthetic continua. 